\title{
Monsel's solution for first degree perineal bleeding tears treatment: a randomized controlled clinical trial
}

\begin{abstract}
Summary
Introduction: During vaginal delivery, tears can occur in different areas of the lower genital tract. The policy of "restrictive episiotomy" increases anterior perineal trauma, mainly paraurethral tears. The topical application of Monsel's solution in first degree perineal bleeding tears may benefit patients because of less use of anesthetics, sutures and procedure time.
\end{abstract}

Objective: To determine if the Monsel's solution stops active bleeding in first degree tears compared to traditional suture.

Materials and methods: Randomized clinical trial, carried out at Hospital Nacional "Prof. Alejandro Posadas" from January 2011 to December 2013. 330 patients with recent postpartum vaginal delivery were admitted with a diagnosis of first degree bleeding tears. They were randomized to receive the application of Monsel's solution, until stopping bleeding (intervention group) or traditional suture (control group). The primary outcome was the stoppage of active bleeding. Secondary outcomes were: patient discomfort with the procedure, practicality of the procedure, alterations in the scarring of the tear and wound infections. Crude and adjusted ORs and 95\% CI were calculated. Analysis was done by intention to treat. The study was approved by the Bioethics Committee of the Hospital.

Results: Monsel's solution was effective for the stoppage of active bleeding in $86.7 \%$ of first degree bleeding tears. Out of 150 patients randomized to the intervention group, 130 met the primary outcome, ORc 0.04 (95\% CI $0.01-0.27) / \mathrm{ORa} 0.05$ (95\% CI $0.01-0.34)$ Monsel's solution was safe, because it did not interfere with the scarring process It also offered a protective aspect on wound infections. The procedure was practical and simple for the intervening professionals and, despite being an irritant for the mucous; it was well tolerated by the patients.

Conclusion: The Monsel's solution appears effective, practical and safe for treatment of first degree bleeding tears.

Keywords: bleeding tears, Monsel's solution, vaginal delivery
Volume II Issue I - 2020

\author{
Casale Roberto Antonio,' Varela Silvana² \\ 'Head of Maternal \& Child Department, Hospital Nacional "Prof. \\ Alejandro Posadas", Argentina \\ 2Methodological Adviser, Epidemiology, Hospital Nacional "Prof. \\ Alejandro Posadas",Argentina
}

\author{
Correspondence: Casale, Roberto Antonio. MD, FACOG, \\ Head of Maternal \& Child Department, Hospital Nacional "Prof. \\ Alejandro Posadas" Buenos Aires, Argentina,
} Email rcasale@intramed.net

Received: January 17, 2020 | Published: February 13, 2020

\section{Introduction}

The use of a "restrictive episiotomy" policy increases anterior perineal trauma, primarily paraurethral tears. ${ }^{1}$ These tears are usually small but they produce important bleeding and should be sutured immediately. The presence of edema and certain conditions of the perineum such as scars, episiotomy or tears from a previous pregnancy, all which interfere with elasticity, are predisposing factors for the development of paraurethral tears. The occipitosacras varieties of fetal presentation in which the fetal head emerges in occiput posterior position, also cause greater perineal distention. ${ }^{2}$

The Monsel solution (ferric subsulfate) is a topical agent used to obtain hemostasis since 1856 described primarily by Leon Monsel (1816-1878). The mechanism of action to achieve hemostasis is the oxidation capacity of the subsulfate group and the agglutination of blood proteins. The solution brought into contact with a small amount of blood triggers coagulation, favors fibrin formation and develops plugs that produce occlusion of the capillary vessels.
A Cochrane review including 7 randomized and quasi-randomized studies $^{3}$ regarding interventions to prevent blood loss during the treatment of intraepithelial cervix neoplasia, reported that the Monsel's solution is more effective in reducing perioperative bleeding and secondary bleeding, than the suture of the wound. This review highlights that the vaginal compress with Monsel's solution located against the cervix appears to be significantly superior to the usual cervix suture. $^{4}$

In 2003, a descriptive study with 50 patients evaluated the efficacy of Monsel's solution in first degree perineal bleeding tears. Only one patient required suturing after the application of Monsel's solution, with an effectiveness of $98 \%$. Scarring was successful in all patients and they did not report pain, except for a slight burning at the time of application. ${ }^{5}$

We developed a randomized clinical trial to evaluate the hypothesis that in cases of first-degree perineal bleeding tears complicating vaginal delivery Monsel's solution is effective to stop active bleeding. 


\section{Methods}

\section{Study design and participants}

We conducted a single center randomized controlled clinical trial, at the Hospital Nacional Prof. A. Posadas, Province of Buenos Aires, Argentina. The institutional review board approved the protocol and consent form. Upon entering the delivery area, those women potentially eligible in labor, were approached and gave written consent. Eligible patients were mothers in the immediate postpartum period who had first degree perineal bleeding tears located in the paraurethral mucosa, vaginal introitus, lips, vulvar forks and/or posterior perineal area, and who after compression with gauze for two minutes over the tear zone continued with active bleeding.

The inclusion of the patients was independent of their age and parity. Women with tears that compromised the perineal muscles, those with vaginal or cervix tears, the patients who presented genital warts and/or varicose veins on external genitals were not included. Anticoagulated patients with heparin, dicumarinics or salicylates and those with blood dyscrasias of different types were also not included.

Following delivery, when the presence of tears was verified, randomization was performed.

\section{Randomization and masking}

Eligible women were randomly assigned in a $1: 1$ ratio to the intervention group (Monsel's solution) or the control group (immediate suture) with computer-generated allocation sequence in block sizes of thirty (created by a statistician who was not involved again in the trial until statistical analysis of the results). Allocation was concealed by sequentially numbered sealed opaque envelopes. The nature of the intervention prevented us from masking the study

\section{Procedures}

In the intervention group (Monsel), the Monsel's solution was applied with a small sterile gauze swab, intermittently, until the hemostasis of the bleeding tear was stopped. If after a period of two minutes blood loss continued, the suture was done with chrome catgut $\# 1$, making simple stitches. The control group was immediately sutured with chrome catgut $\# 1$, making simple stitches as well (Figure 1).

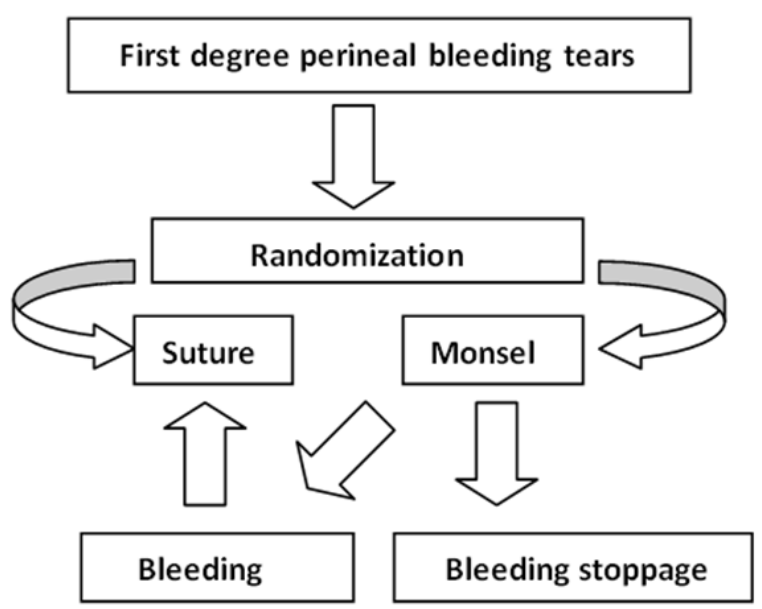

Figure I Process.
Patients were evaluated at two and at 48 hours postpartum, before discharge from the hospital and again at seven days at the outpatients clinic. All women admitted received the usual postpartum controls. Those admitted to the "intervention group" had no different postpartum care than those admitted to the "control group" in terms of rest, perineal care and use of antiseptics.

The main outcome variable was the stoppage of active bleeding. Secondary outcome variables were: Discomfort with the procedure, practicality, alterations in the scarring of the tear and the appearance of wound infection.

The sensation of discomfort, specifically reported by the patients as: burning, discomfort or pain at the site of bleeding was evaluated immediately, at 2 hours, 48 hours and in the postpartum control by office.

Practicality was reported by the professional who performed the delivery at the end of the procedure by answering the question: "Were the application of Monsel's solution or the suture practical or did it represent any degree of difficulty?"

The presence of alterations of the wound scarring such as dehiscence due to separation of the edges or necrosis of the wound were evaluated in the postpartum within 48 hours of the procedure and during the post partum outpatients' visits.

The development of signs of infection, such as phlogosis or suppuration of the wound, were evaluated at the patient's room at 48 hours after delivery and at the time of concurrence to control of the outpatients office.

\section{Statistical analysis}

Categorical variables were presented as frequency measures and compared using Fisher/chi2 exact test. Continuous variables were presented as central tendency and their dispersions (mean and standard deviation), making comparisons through the T-Test.

The magnitude of the main effect was measured in OR, with its 95\% Confidence Intervals. Likewise, statistical adjustments were made through a multiple logistic regression models where the presence of potential confounders was considered (educational level, maternal age, parity, gestational age, history of episiotomy or tears in previous deliveries, maternal pathology, prenatal control and episiotomy in current delivery) presenting their respective adjusted OR with $95 \%$ CI. The statistical analysis was carried out by "intention to treat".

The sample size was calculated based on data obtained in the Perinatal Computerized System with a prevalence of first-degree tears requiring sutures of $26.6 \%$. To find a statistical significance, a power of $80 \%$ was considered, with an error $\alpha=0.05$, aiming to reduce the incidence of sutures by $50 \%$ and allowing a loss in follow-up of $10 \%$. The minimum sample necessary to find the desired effect was 300 patients. (150/group)

The EPI Info program version 3.5.3 2011 and STATA 11.1 were used for the analysis. 2011

\section{Results}

From May 2011 to October 2013, 9431 births were registered. 520 women were potentially eligible, of which 330 met the inclusion criteria (Figure 2) (Table 1) (Table 2). 


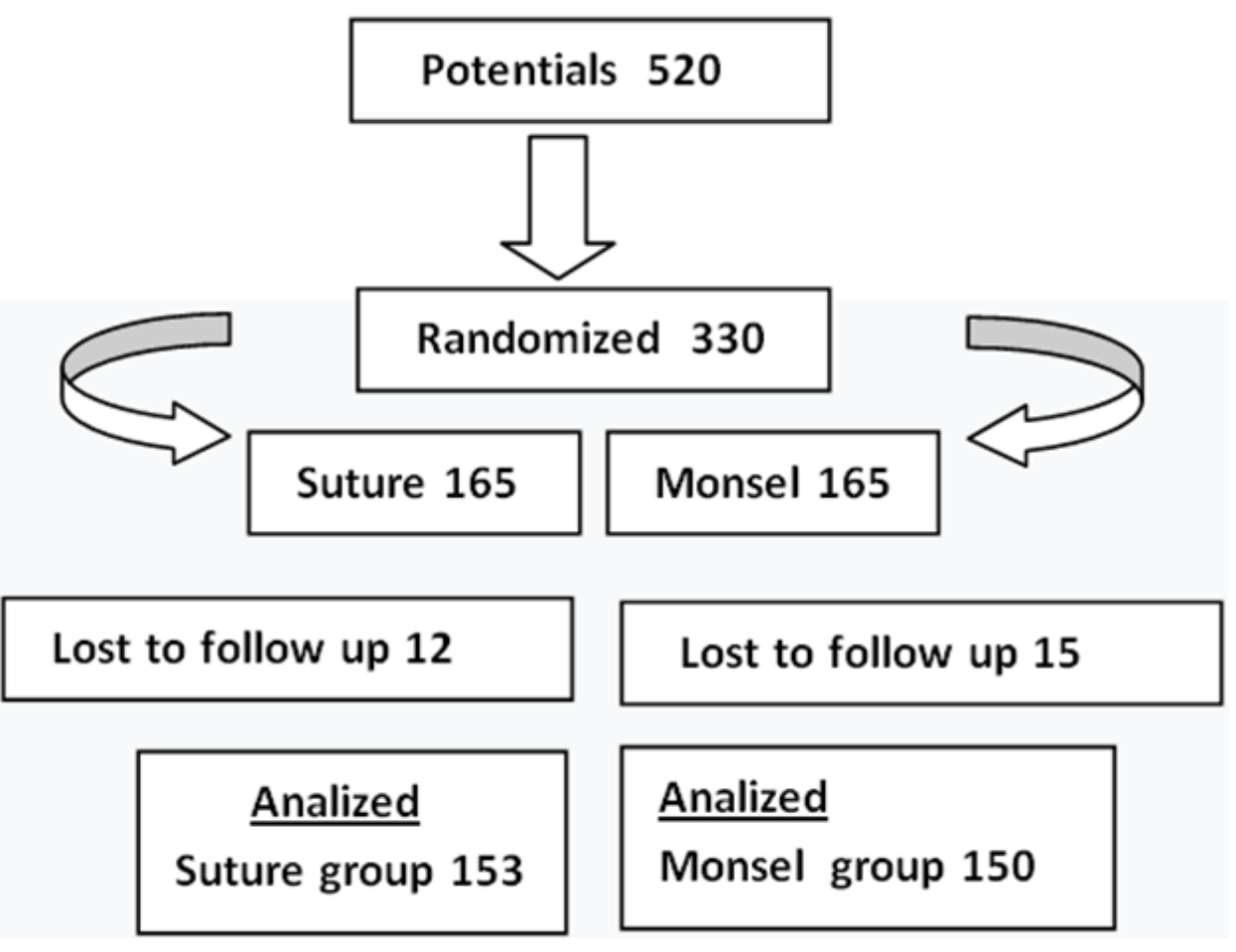

Figure 2 Randomization and Follow up.

Table I Characteristics of the groups

\begin{tabular}{|c|c|c|c|}
\hline Variables & Monsel $(\mathrm{N}=\mid 50)$ & Suture $(N=153)$ & $P$ valor \\
\hline Media (DS) / Frecuency (\%) & $(n / N)$ & $(n / N)$ & \\
\hline Age & $24(6.1)$ & $24(1.1)$ & $0.77^{*}$ \\
\hline Pregnancy & $2.3(1.3)$ & $2.2(1.2)$ & $0.28 *$ \\
\hline Deliveries & $2.1(1.2)$ & $1.9(1.0)$ & $0.09 *$ \\
\hline No delivered & $33 \%(50 / 150)$ & $34 \%(53 / 153)$ & $0.50 * *$ \\
\hline One or two delivered & $48 \%(72 / 150)$ & $52 \%(79 / 153)$ & \\
\hline Three o more & $19 \%(28 / 150)$ & $14 \%(2|/| 53)$ & \\
\hline \multicolumn{4}{|l|}{ Educational Level } \\
\hline None & $0.6 \%(1 / 148)$ & $0.6 \%(1 / 152)$ & $0.58^{* * *}$ \\
\hline Elementary School & $40 \%(59 / 148)$ & $43 \%(65 / 152)$ & \\
\hline High School & $57 \%(84 / 148)$ & $56 \%(85 / 152)$ & \\
\hline University & $2.4 \%(4 / 148)$ & $0.4 \%(1 / 152)$ & \\
\hline \multicolumn{4}{|l|}{ Background } \\
\hline Episiotomy & $52 \%(78 / 150)$ & $43 \%(66 / 153)$ & $0.12^{* *}$ \\
\hline Tears & $19 \%(29 / 150)$ & $19 \%(30 / 153)$ & $0.95^{* *}$ \\
\hline
\end{tabular}

*Test Student

**Chi 2

***Fisher exact test 
Table 2 Characteristics of pregnancy and delivery

\begin{tabular}{|c|c|c|c|}
\hline Variables & Monsel $(N=\mid 50)$ & Suture $(N=153)$ & $\mathbf{P}$ valor \\
\hline Media (SD)/ Frecuency (\%) & $(\mathbf{n} / \mathbf{N})$ & $(n / N)$ & \\
\hline Prenatal controls & $6.9(2.3)$ & $7(2.3)$ & $0.7 I^{*}$ \\
\hline \multicolumn{4}{|l|}{ Delivery } \\
\hline Spontaneus & $92 \%(137 / 149)$ & $95 \%(145 / 153)$ & $0.32 * *$ \\
\hline Induced & $8 \%(12 / 149)$ & $5 \%(8 / 153)$ & \\
\hline Time of delivery (hours) & 3.04 (DS I.66) & 3.17 (DS I.84) & $0.52 *$ \\
\hline Episiotomy & $14 \%(20 / \mid 43)$ & $17 \%(23 / 137)$ & $0.5 \mathrm{I}^{* *}$ \\
\hline Complications & $3 \%(4 / 148)$ & $2 \%(3 / 153)$ & $0.7 \mid * * *$ \\
\hline
\end{tabular}

*Test Student

***hi 2

****Fisher exact test

Monsel's solution was effective to stop active bleeding in 130/150 tears crude OR 0.04 (95\% CI 0.01-0.27) and adjusted OR 0.05 (95\% patients (86.7\%, CI 80.2-91.7) with first-degree perineal bleeding CI 0.01-0.34) (Table 3) (Figure 3).

Table 3 Main outcome

\begin{tabular}{lllll}
\hline Stoppage of bleeding & Monsel $(\mathbf{N}=150)$ & Suture $(\mathbf{N}=153)$ & OR Crude & OR Adjusted* \\
\hline$(n / N)$ & $(n / N)$ & IC $95 \%$ & IC $95 \%$ \\
$130 / 150$ & $152 / 153$ & OR 0.04 & OR 0.05 \\
& & IC $95 \% 0.01-0.27$ & IC $95 \% 0.01-0.34$ &
\end{tabular}

*Adjusted for educational level, maternal age, number of deliveries, previous episiotomy or tears, maternal complications, prenatal control and current episiotomy.

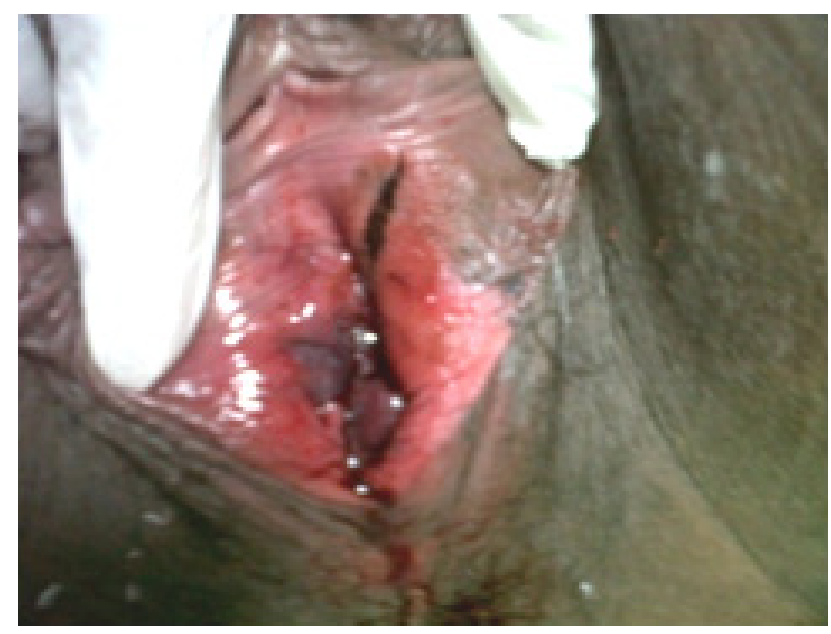

Figure 3 Stoppage of bleeding.

An additional suture had to be performed for the resolution of bleeding in 20 patients (13.3\%, CI 8.3-19.8), in the Monsel's group.

There were no differences between the patients of the Monsel group and the control group in need of suture at the evaluation two hours after the procedure $(\mathrm{p}=0.36)$ (Table 4$)$.
Table 4 Assessment

\begin{tabular}{llll}
\hline & $\begin{array}{l}\text { Monsel }(\mathbf{N}=150) \\
(\mathbf{n} / \mathbf{N})\end{array}$ & $\begin{array}{l}\text { Suture }(\mathbf{N}=153) \\
(\mathbf{n} / \mathbf{N})\end{array}$ & P valor \\
\hline $\begin{array}{l}\text { Bleeding } 2 \text { hs } \\
\text { Discomfort or } \\
\text { pain }\end{array}$ & $3 / 150$ & $1 / 153$ & $0.36^{*}$ \\
2 hs & $38 / 150$ & $33 / 152$ & $0.45^{* *}$ \\
48 hs & $6 / 149$ & $12 / 153$ & $0.16^{* *}$ \\
7 días & $1 / 149$ & $12 / 153$ & $0.003^{*}$ \\
$\begin{array}{l}\text { Scar disorder } \\
48 \text { hs. }\end{array}$ & $2 / 119$ & $6 / 101$ & $0.09 *$ \\
$\begin{array}{l}\text { Scar disorder } 7 \\
\text { days }\end{array}$ & $0 / 150$ & $1 / 153$ & $1 *$ \\
$\begin{array}{l}\text { Wound infection } \\
48 \text { hs. }\end{array}$ & $0 / 150$ & $9 / 101$ & $0.006^{*}$ \\
$\begin{array}{l}\text { Wound infection } \\
7 \text { days }\end{array}$ & $1 / 119$ & $2 / 153$ & $0.50^{*}$ \\
\hline
\end{tabular}

*Fisher Exact Test

**Test chi2 
Immediately and at 2 hours after treatment, there was no difference in discomfort with the procedure (burning, discomfort or pain in the site of bleeding) between the groups $(\mathrm{p}=0.45, \mathrm{p}=0.16)$ (Figure 4). However, patient comfort was significantly improving over time and after 48 hours of the procedure only $0.7 \%$ of the Monsel Group and $7.8 \%$ of the Suture Group reported some type of discomfort $(\mathrm{p}=0.003)$. At the follow up outpatient clinic, 119 patients from the Monsel Group and 101 from the Suture Group were evaluated. Two patients of the Monsel Group and six of the Suture Group continued to present some discomfort (Table 4).

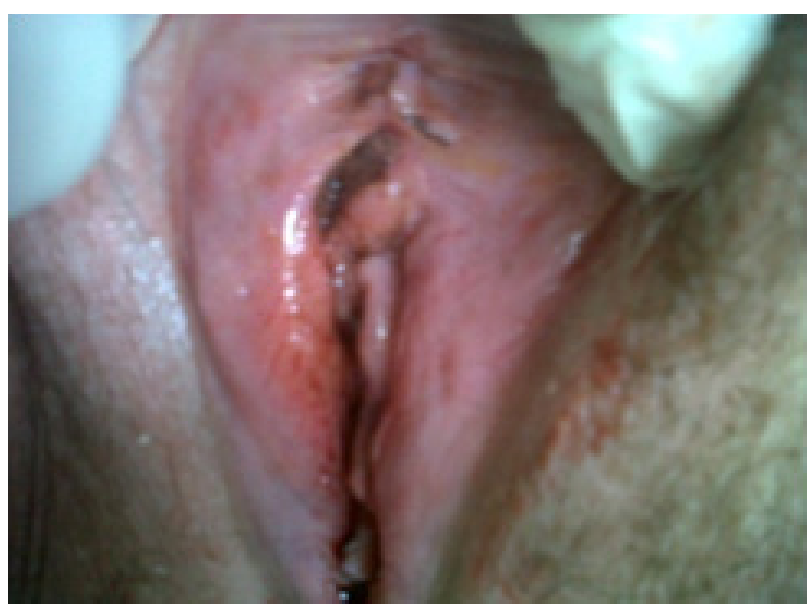

Figure 4 Wound scarring 2 hs.

At 48 hours, no patient of the Monsel Group presented scar disorder. A patient from the Suture Group $(0.7 \%)$ presented separation of the wound edges. In the postpartum controls, scar disorders were detected in one patient $(0.7 \%)$ of the Monsel Group and in nine patients $(8.8 \%)$ of the Suture Group.

At 48 hours after delivery, no patient of the Monsel Group showed signs of infection. Two patients $(1.8 \%)$ of the Suture Group had an infection. In the outpatient control, signs of infection were detected in one patient $(0.8 \%)$ of the Monsel Group and in seven patients $(6.9 \%)$ of the Suture Group (Table 4) (Figure 5).

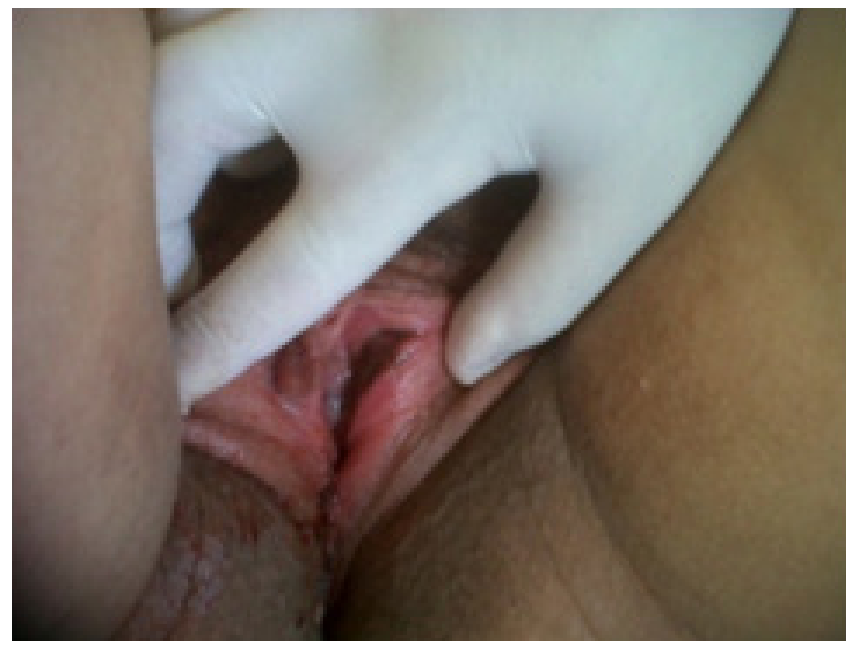

Figure 5 Wound scarring $48 \mathrm{hs}$.
About the practicality of the procedure, in view of the professional's perception, $93.3 \%$ of the ones who applied the intervention reported it was easy and practical. ${ }^{6-12}$

\section{Discussion}

The application of the Monsel's solution was effective for the stoppage of active bleeding in $86.7 \%$ of patients with first-degree perineal bleeding tears. Out of 150 patients who received the new procedure; in 130 the success was confirmed, accepting the hypothesis that the treatment would prevent sutures, in at least $50 \%$ of the patients. The result was highly significant with a crude OR of 0.04 (95\% CI 0.01-0.27) and adjusted of 0.05 (95\% CI 0.01-0.34).

The technique is very simple and does not need any additional training. The solution is applied intermittently into the tear and, in contact with the blood, coagulates the proteins. Thus, the appearance of a black paste in the wound that achieves the hemostatic effect was observed. The application of Monsel's solution was practical and easy in $93.3 \%$ of the procedures performed. ${ }^{13-20}$

As the Monsel's solution is a protein coagulant agent with a certain causticity, it was important to evaluate its safety and whether it damaged the tissues or interfered with the subsequent scarring process. The Monsel's solution did not interfere with the scarring process and also offered a protective action over wound infections. At 48 hours of the procedure, no patient of the Monsel Group had alterations in scarring, specifically necrosis due to the application of the solution. On the other hand, in the Suture Group, scar disorders were found in one patient $(0.7 \%)$. Signs of infection, such as inflammation or wound suppuration, were detected in one patient $(0.8 \%)$ of the Monsel Group and in seven patients $(6.9 \%)$ of the Suture Group during postpartum control. These results suggest some potential bactericidal effect of the Monsel solution.

From another point of view, it was important to know if the application of the Monsel's solution caused a sensation of burning, discomfort or pain at the application site. A qualitative survey of patients of both groups was performed. In the Monsel Group 25.3\% of patients reported some discomfort immediately after the procedure compared with $21.5 \%$ in the Suture Group. This "non-significant" difference $(\mathrm{p} 0.45)$ reveals that both procedures generate discomfort and dissatisfaction. It was observed that the discomfort, which is produced by the irritation that the Solution generates at the application site, decreases over the course of the days. It appears that over time Monsel's solution is better tolerated tan the stiches. ${ }^{21-24}$

It was shown that the application of the Monsel Solution was effective for the stoppage of active bleeding of patients with firstdegree bleeding perineal tears, confirming the hypothesis that the treatment would prevent sutures in at least $50 \%$ of the patients.

The Monsel's solution had lower adverse effects related to wound scarring, lower infection rates in the postpartum controls and without interference with the scarring process (Figures 6-8).

The patients did not present necrosis at the application site; and also, due to its bactericidal power, It provides a protective effect on the development of infections in the treated tears.

As demonstrated in this research, the Monsel's solution is effective, practical and safe for the treatment of first-degree bleeding perineal 
tears. The procedure can be recommended in clinical practice. We suggest that in the presence of a first degree bleeding perineal tear, Monsel's solution could be used in the first instance and in cases of persistence of active bleeding, traditional suture should be used.

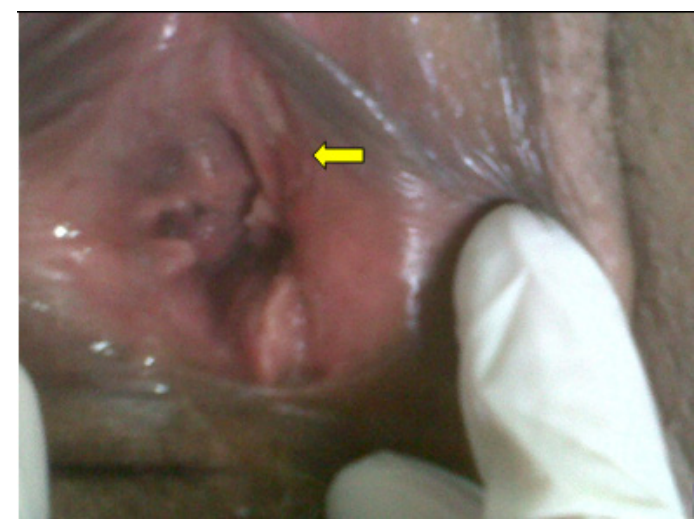

Figure 6 Paraurethral scarring.

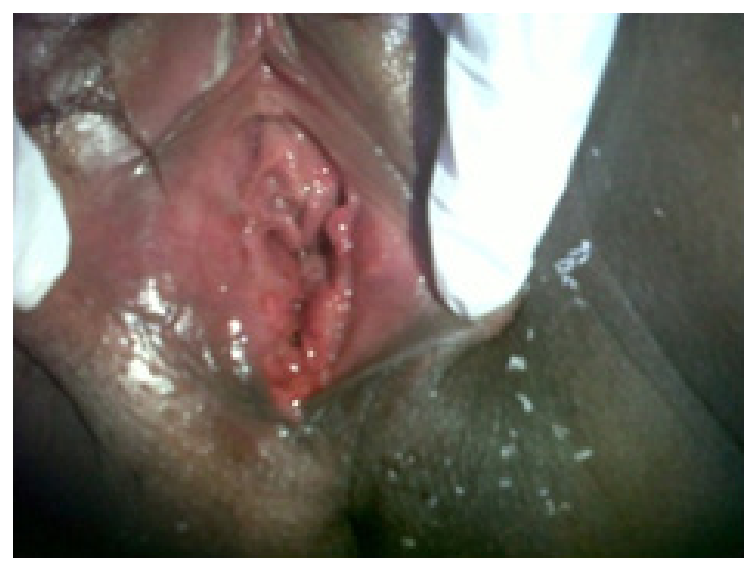

Figure 7 Complete scarring.

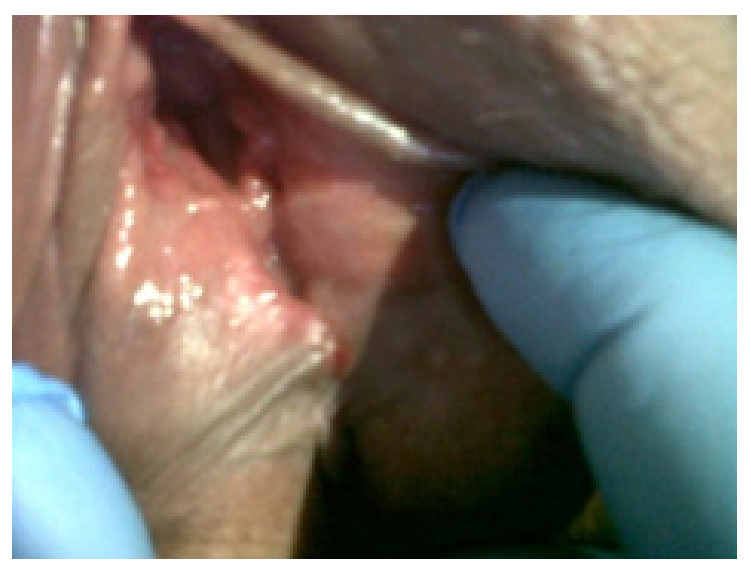

Figure 8 Complete scarring 6hs.

\section{Acknowledgments}

We wish to thank Ferreiros Alberto, Bamundi Cecilia; Barthou Soledad; Botta Maria; Burgos Marianela; Caivano Marianela; Cutolo Gisela; Fanti Valeria; Kosiuk Gisela; GutierrezErica; Gutierrez
Carlos; Grijalba Chiara; Grijalba Nancy; Gonzalez Ivana; Lauphan Vanina; Lombardo Jésica; Losardo Milena; Manino Joaquin; Martinez Federico; Navarro Romina; Otero Paula; Pascale Julian; Prado Laura; Peri Cruzado Diana; Ponti Alejandro; Quintero Hoyos Yarle; Rodriguez Carolina; Rodriguez Aldáz Camila; San Emeterio Susana; Sandjian Maria; Viberti Gisela; Vilo Paola; Vivas Aldana; Vuirli Denise; Yuricich Noelia; Zagasti Julia.

Reviewer: Professor Dr. Nestor Vain.

\section{Funding}

None.

\section{Conflicts of interest}

The authors declare there are no conflicts of interest.

\section{References}

1. Carroli G, Belizan J. Episiotomy in vaginal delivery (Cochrane Review). Oxford: The Cochrane Library; 2002.

2. Schwarcz R, Duverges C, Gonzalo Diaz A, et al. Obstetrics. 5th ed. El Ateneo; 2003

3. Martin-Hirsch PL, Kitchener H. Interventions for the prevention of blood loss during the treatment of intraepithelial cervical neoplasia. Reproduction of a Cochrane review. 2008.

4. Gilbert L, Saunders N, Stringer R, et al. Hemostasis and cold knife cone biopsy: a prospective randomised trial comparing a suture versus nonsuture technique. Obstet Gynecol. 1989;74(4):640-643.

5. Ferreirós A, Marra K, Murilla V, et al. Is the Monsel Solution useful for achieving hemostasis and healing of first-degree tears postpartum bleeding? Obstetrics Service Hospital Nac. Professor A Inns Haedo Prov, Buenos Aires. Argentina: Abstracs FASGO Congress; 2003.

6. Armstrong RB, Nichols J, Pachance J. Punch biopsy wounds treated with Monsel Solution, on a collagen matrics: a comparison of healing. Arch Dermatopath. 1986;122(5):546-549.

7. Attarbashi S, Faulkner RL, Slade RJ. The use of Monsel's solution and vaginal pack for haemostasis in cold knife cone biopsy. J Obstet Gynaecol. 2007;27(2):189.

8. Casale RA, Franze FC, Ferreiros JA, et al. Cervical pregnancy of the second trimester. Conservative surgical technique Cervicotomy by abdominal route. Original work FASGO Magazine. 2004;3(1):22-26.

9. Chumnan K, Jatupol S, Prapaporn S, et al. Routine prophylactic application of Monsel's solution after loop electrosurgical excision procedure of the cervix: Is it necessary? J Obstet Gynaecol Res. 2007;33(3):299-304.

10. Stewart D, Lisa R, Rami A. The use of intrauterine Monsel's solution in severe hemorrhage after evacuation of retained products of conception: a case report. Am J Obstet Gynecol. 2007;196(2):e6-e7.

11. Flemming N. Can the suturing methods make a difference in postpartum perineal pain? J Nurse Midwifery. 1990;35(1):19-25.

12. Garrett Audrey P, Wenham RM, Sheets EE. Monsel's solution: a brief history. Journal of Lower Genital Tract Disease. 2002;6(4):225-227.

13. Guyatt. Updated by the center of evidence based medicine. JAMA. $1995 ; 274: 1800$

14. Grant A, Gordon B, Mackrodat C, et al. The Ipswich childbirth study: one year follow up of alternative methods used in perineal repair. BJOG. 2001;108(1):34-40. 
15. Jetmore AB, Heryer JW, Conner WE. Monsel's solution: a kinder, gentler hemostatic. Dis Colon Rectum. 1993;36(9):866-867.

16. Francisco LJ, Gregorio TO, Gervasio AL. Manual of evidence-based medicine. México: El Manual Moderno; 2001. 250 p.

17. Mark ER, Sharon JM, Paul DF, et al. Monsel's solution: a potential vector for nosocomial infection? Infect Control Hosp Epidemiol. 2003;24(2):142-144.

18. Monsel Solution. Technical information: monsel solution, ferric subsulfate solution. Gordon Laboratorie; 1996.

19. Nassif JC, Keklikián RI. Obstetrics, Fundamentals and practical approach Editorial Panamericana; 2012.
20. Shuhaiber JH, Lipnick S, Teresi M. More on Monsel Solution. Surgery. 2005;137(2):263-264.

21. Spitzer M, Chernys HE. Monsel solution induced artefact in the uterine cervix. Am J Obstet Gynecol. 1996;175(5):1204-1207.

22. Tam. KF, Lee TP, Ngan HY. Hemostasis following cervical punch biopsy using Monsel's solution. Int J Gynaecol Obstet. 2005;88(2):160-161.

23. Walfisch A, Hallak M, Harlev S, et al. Association of spontaneous perineal stretching during delivery with perineal lacerations. Israel J Reprod Med. 2005;50(1):23-28.

24. Cunningham FG. Williams Obstetrics. 22nd ed. Interamerican of Mexico; 2006 . 